\title{
Nonsteady frictional heating on a sliding contact
}

\author{
Vladimir B. Zelentsov, and Boris I. Mitrin* \\ Research and Education Center "Materials", Don State Technical University, 344000 Rostov-on-Don, \\ Russia
}

\begin{abstract}
We consider quasi-static contact problem on frictional heating on a sliding contact of a rotating rigid cylinder and a half-plane. The cylinder is pressed towards the half-plane material. The problem is reduced to solution of a singular integral equation with respect to contact stresses. Solution of the singular equation is looked for in a class of functions limited on the edge, with two additional conditions to determine timedependent boundaries of the contact area. Temperature at the contact and inside the half-plane is determined in terms of contact stresses.
\end{abstract}

\section{Introduction}

In numerous industries ranging from metallurgy to microelectronics, mechanical manufacturing processes, such as cutting, drilling, grinding, polishing and others, involve frictional heating. Heating of bodies subjected to such operations negatively affect morphology of their surfaces. This is especially true in high-speed machining operations and gives rise to the need in control of temperature during mechanical processing.

Mathematical modeling of the frictional contact can give relationship between temperature and properties of the machined material, speed of machining, shape of the instrument and other factors. It allows to optimize machining process and to create necessary conditions for precise design of shape and topography of a product.

Problem of frictional sliding of a punch with frictional heat generation was previously considered by some researchers. Barber [1] proposed some steady-state formulations for axisymmetric and two-dimensional problems involving frictional heating. Axisymmetric steady-state problems was further considered in works of Yevtushenko and KulchytskyZhyhailo [2]. Particular interest is paid to thermoelastic contact involving functionally graded materials. Krenev et al [3] considered axisymmetric problem of a heated punch acting on a functionally graded half-space using semi-analytical method. Choi and Paulino [4] derived an analytical solution to the problem of sliding of a flat punch over the strip on an elastic half-plane with an exponentially functionally graded interlayer between them. Particular case of a functionally graded strip on an elastic half-plane was considered using the similar technique by Chen and Chen in [5]. Recently, frictional contact problem between a rigid conducting flat punch a functionally graded magnetoelectroelastic layer [6] or half-plane [7] with frictional heat generation was considered.

\footnotetext{
* Corresponding author: bmitrin@dstu.edu.ru
} 
In the works listed above, horizontal velocity of the punch was assumed to be very low, so only steady-state sliding was considered. In this paper, we propose an approach to deal with transient problem, which allow one to study time dependence of stress, temperature, and displacements inside the material during frictional sliding process.

\section{Problem statement}

We consider a quasi-static problem about frictional heating on a sliding contact during indentation of a circular cylinder with radius $R$ into the half-plane material. The cylinder rotates with angular velocity $\omega$. Boundary conditions of this contact problem formulated in the framework of thermoelasticity theory are as follows:

$$
\begin{array}{lll}
y=0 & v(x, 0, t)=f(x, t) & x \in(a, b) \\
t>0 & \sigma_{x y}(x, 0, t)=f \sigma_{y y}(x, 0, t) & x \in(a, b) \\
& K \frac{\partial}{\partial y} T(x, 0, t)=-f V \sigma_{y y}(x, 0, t) & x \in(a, b) \\
& \sigma_{y y}(x, 0, t)=\sigma_{x y}(x, 0, t)=K \frac{\partial}{\partial t} T(x, 0, t)=0 & x \notin(a, b)
\end{array}
$$

where $\sigma_{y y}(x, y, t), \sigma_{x y}(x, y, t)$ are normal and tangential stresses, $u(x, y, t), v(x, y, t)$ are horizontal and vertical displacements, correspondingly, $a=a(t), b=b(t)$ are initially not known boundaries of the contact area, $T(x, y, t)$ is distribution of temperature in the half-plane material, $f(x, t)$ determines shape of the cylinder base and immersion in the half-plane, $f$ is friction coefficient, $V=\omega R$ is linear velocity of the cylinder, $K$ is heat conductivity of the half-plane material. Boundary condition (3) means that tangential stresses $\sigma_{x y}$ at the contact interface are proportional to normal stresses $\sigma_{y y}$ with a factor $f$. Boundary condition (4) means that all the heat generated at the contact interface flows into the half-plane material.

Normal and tangential stresses are determined from [8]

$$
\begin{gathered}
\sigma_{y y}=(\lambda+2 \mu) \frac{\partial v}{\partial y}+\lambda \frac{\partial u}{\partial x}-(3 \lambda+2 \mu) \alpha_{T}\left(T-T_{0}\right) \\
\sigma_{x y}=\mu\left(\frac{\partial u}{\partial y}+\frac{\partial v}{\partial x}\right)
\end{gathered}
$$

Displacements $u(x, y, t), v(x, y, t)$ of the half-plane vanishes at infinity $\sqrt{x^{2}+y^{2}} \rightarrow \infty$.

Initial conditions on displacements and their rates are zero

$$
u(x, y, 0)=v(x, y, 0)=\dot{u}(x, y, 0)=\dot{v}(x, y, 0)=0 \quad|x|<\infty, y \leq 0
$$

while initial temperature in the half-plane is equal to $T_{0}$

$$
T(x, y, 0)=T_{0} \quad|x|<\infty, y \leq 0
$$

Differential equations of thermoelasticity governing behavior of the half-plane material in the quasi-static case have the form

$$
\begin{aligned}
& \mu \Delta u+(\lambda+\mu) \frac{\partial \theta}{\partial x}=(3 \lambda+2 \mu) \alpha_{T} \frac{\partial T}{\partial x} \\
& \mu \Delta v+(\lambda+\mu) \frac{\partial \theta}{\partial y}=(3 \lambda+2 \mu) \alpha_{T} \frac{\partial T}{\partial y}
\end{aligned} \quad \theta=\frac{\partial u}{\partial x}+\frac{\partial v}{\partial y}
$$


where $\mu, \lambda$ are Lamé parameters, $\alpha_{T}$ is linear heat expansion coefficient of the half-plane material, $\Delta$ is the Laplace operator.

Temperature distribution $T(x, y, t)$ in the half-plane is governed by the heat equation

$$
\Delta T-\frac{1}{\kappa} \frac{\partial}{\partial t} T(x, y, t)=0
$$

where $\kappa$ is thermal diffusivity of the half-plane material.

\section{Method of the integral equation solution}

To solve the quasi-static contact problem (1)-(8), we used the Laplace (with respect to time $t$ ) and Fourier (with respect to longitudinal coordinate $x$ ) integral transforms. After applying the transforms, the problem solution is reduced to the integral equation with a logarithmic singularity

$$
\begin{gathered}
\int_{0}^{t} d \tau \int_{a(\tau)}^{b(\tau)} \varphi(\xi, \tau) k(\xi-x, t-\tau) d \xi=2 \pi \frac{\lambda+\mu}{\lambda+2 \mu} 2 \mu f(x, t) \quad x \in(a, b) \\
k(u, v)=\frac{1}{2 \pi i} \int_{-i \infty}^{i \infty} e^{p v} d p \int_{-\infty}^{\infty} K(\alpha, p) e^{i \alpha u} d \alpha \\
K(\alpha, p)=\frac{1}{|\alpha|}+\frac{\mu}{\lambda+2 \mu} f \frac{1}{i \alpha}+\frac{\hat{V}}{\eta} G(\alpha, p) \\
G(\alpha, p)=\frac{|\alpha|}{\sigma(\alpha, p)\left(|\alpha| \sigma(\alpha, p)+\alpha^{2}\right)}, \quad \sigma(\alpha, p)=\sqrt{\alpha^{2}+\frac{p}{\kappa}} \\
\hat{V}=\frac{f V \alpha_{T}(3 \lambda+2 \mu)}{K}, \quad \eta=\frac{\lambda+2 \mu}{2 \mu}
\end{gathered}
$$

Integrals in (10) are understood in generalized meaning [9].

\section{Problem solution}

Calculating dual integral (10) of first two terms in (11), we get

$$
\begin{gathered}
k(\xi-x, t-\tau)=-2 \ln |\xi-x|-2 \gamma+\pi \frac{\mu}{\lambda+2 \mu} f \operatorname{sgn}(\xi-x)+r(\xi-x, t-\tau) \\
r(u, v)=\frac{\hat{V}}{\eta} \frac{1}{2 \pi i} \int_{-i \infty}^{i \infty} e^{p v} d p \int_{-\infty}^{\infty} G(\alpha, p) e^{i \alpha u} d \alpha
\end{gathered}
$$

Substituting (12) to (9) and differentiating left and right-hand sides of the obtained equation with respect to $x$, we obtain a singular integral equation

$$
\begin{gathered}
-\frac{\pi}{2} \frac{\mu f}{\lambda+2 \mu} \varphi(x, t)+\int_{0}^{t} d \tau \int_{a(\tau)}^{b(\tau)} \frac{\varphi(\xi, \tau)}{\xi-x} d \xi= \\
\pi \frac{(\lambda+\mu) \mu}{\lambda+2 \mu} f_{x}^{\prime}(x, t)+F_{x}^{\prime}(x, t)
\end{gathered} \quad x \in(a, b)
$$


where

$$
F(x, t)=\int_{0}^{t} d \tau \int_{a(\tau)}^{b(\tau)} \varphi(\xi, \tau) r(x-\xi, t-\tau) d \xi
$$

Solution $\varphi(x, t)$ of the integral equation (13) is constructed [10] in class of functions which are limited on the edges of the integration interval

$$
\varphi(x, t)=\omega(x, t) \sqrt{(x-a(t))(b(t)-x)} \quad \omega(x, t) \in C_{(a, b)}
$$

with two additional conditions from which unknown $a=a(t)$ and $b=b(t)$ are determined.

Temperature at the contact interface and in the half-plane material is determined in terms of contact stresses $\varphi(x, t)$ by a formula

$$
T(x, y, t)-T_{0}=-\frac{f V}{K} \cdot \frac{1}{2 \pi} \int_{0}^{t} \frac{d \tau}{t-\tau} \int_{a(\tau)}^{b(\tau)} \varphi(\xi, \tau) \exp \left(-\frac{y^{2}+(\xi-x)^{2}}{4 \kappa(t-\tau)}\right) d \xi
$$

The obtained formulas allow to calculate temperature at the contact and inside the halfplane material.

This work was supported by the State Assignment of the Ministry of Education and Science of Russia no. 9.1481.2017/4.6 and Russian Foundation for Basic Research grants no. 16-07-00929-a, 17-0701376-a.

\section{References}

1. J.R. Barber, Quart. J. Mech. Appl. Math., 29(1), 1 (1976)

2. A.A. Yevtushenko, R.D. Kulchytsky-Zhyhailo, J. Mech. Phys. Sol., 43(4), 599 (1995)

3. L.I. Krenev, S.M. Aizikovich, Y.V. Tokovyy, Y.C. Wang, Int. J. Sol. Struct., 59, 18 (2015)

4. H.J. Choi, G.H. Paulino, J. Mech. Phys. Sol., 56(4), 1673 (2008)

5. P. Chen, S. Chen, Int. J. Sol. Struct., 50(7-8), 1108 (2013)

6. J. Ma, S. El-Borgi, L.-L. Ke, Y.-S. Wang, J. Therm. Stress., 39(3), 245 (2016)

7. R. Elloumi, S. El-Borgi, M.A. Guler, I. Kallel-Kamoun, Acta Mech., 227, 1123 (2016)

8. A.D. Kovalenko, Introduction to Thermoelasticity (Naukova Dumka, Kiev, 1965)

9. Yu.A. Brychkov, A.P. Prudnikov, Integral Transforms of Generalized Functions (Gordon \& Breach Science Publishers / CRC Press, New York-London, 1989)

10. N.I. Muskhelishvili, D.A. Kveselava, Proc. Tbilisi Math. Inst. Acad. Sci. Georgian SSR, 11, 141 (1942) 